\title{
SN 1987A AND ITS ROLE IN THE LMC
}

\author{
I.J. DANZIGER, P.BOUCHET, C. GOUIFFES, L.B. LUCY \\ European Southern Observatory \\ Karl-Schwarzschild-Str.2 \\ D-8046 Garching bei München, F.R.Germany
}

\begin{abstract}
A brief review is presented of results obtained at ESO on dust formation, evolution of line profiles and luminosities, and chemical abundances including the radioactive species. The most recent evolution of the bolometric light curve is discussed..
\end{abstract}

\section{Dust}

Before the occurrence of SN 1987A, Dwek (1983) and others had conjectured and predicted that dust might form in supernova envelopes at a relatively early phase following the explosion. This was partly prompted by the growing realisation that dust was forming in nova envelopes at fairly high temperatures. Nevertheless, when some of the above authors examined the nature of IR excesses observed in supernovae such as 1979c, 1980k, 1982e it seemed difficult to decide conclusively between models of dust in the envelope and heated external dust causing an IR echo. In the case of $1982 \mathrm{~g}$ Graham et al. (1983) were more positive that the observed phenomenon was the result of an IR echo.

The data available at the time for all of these supernovae were fragmentary, in contrast to the situation for SN 1987A. Spectroscopic evidence for the formation of dust in the envelope of SN 1987A is conclusive. Fig. 1 shows the initial result reported by Danziger et al. (1989) and analysed in detail by Lucy et al. $(1989,1990)$ where the blueward shift of the emission line peaks is evidence for dust formation. This work also analysed the implications of the IR excess observed out to $20 \mu$ (Q band) which developed after day 530; it showed that to be consistent with the bolometric light curve powered by radioactive decay of ${ }^{56} \mathrm{Co}$, this IR excess had to be included in the energy budget, whereas it would have to be excluded if an IR echo was involved. This IR excess results from absorption of radiation at optical wavelengths and its subsequent thermal reemission at the ambient temperature of the dust in the dilute radiation field pervading the envelope. Subsequent analysis of the light curve over a longer period by Suntzeff and Bouchet (1990) has significantly reinforced these conclusions.

It is worth recalling here that the analysis by Lucy et al. (1990) of evidence for a combination of a wavelength dependent and independent extinction based both on behaviour of line profiles and on photometric effects leads to a model of dust distributed in two forms in the envelope - an amorphous distribution of small particles (probably silicates) and a random distribution of optically thick large clumps or clouds which provide geometric obscuration.

In spite of the assertion above concerning the value of spectroscopic criteria, very accurate photometry such as the Geneva system V band made at closely spaced intervals (Fig.2) and taken from the work of Burki et al. (1990) can provide information on the dust forming process, particularly when one is alerted about what to look for. The three vertical lines mark the times 


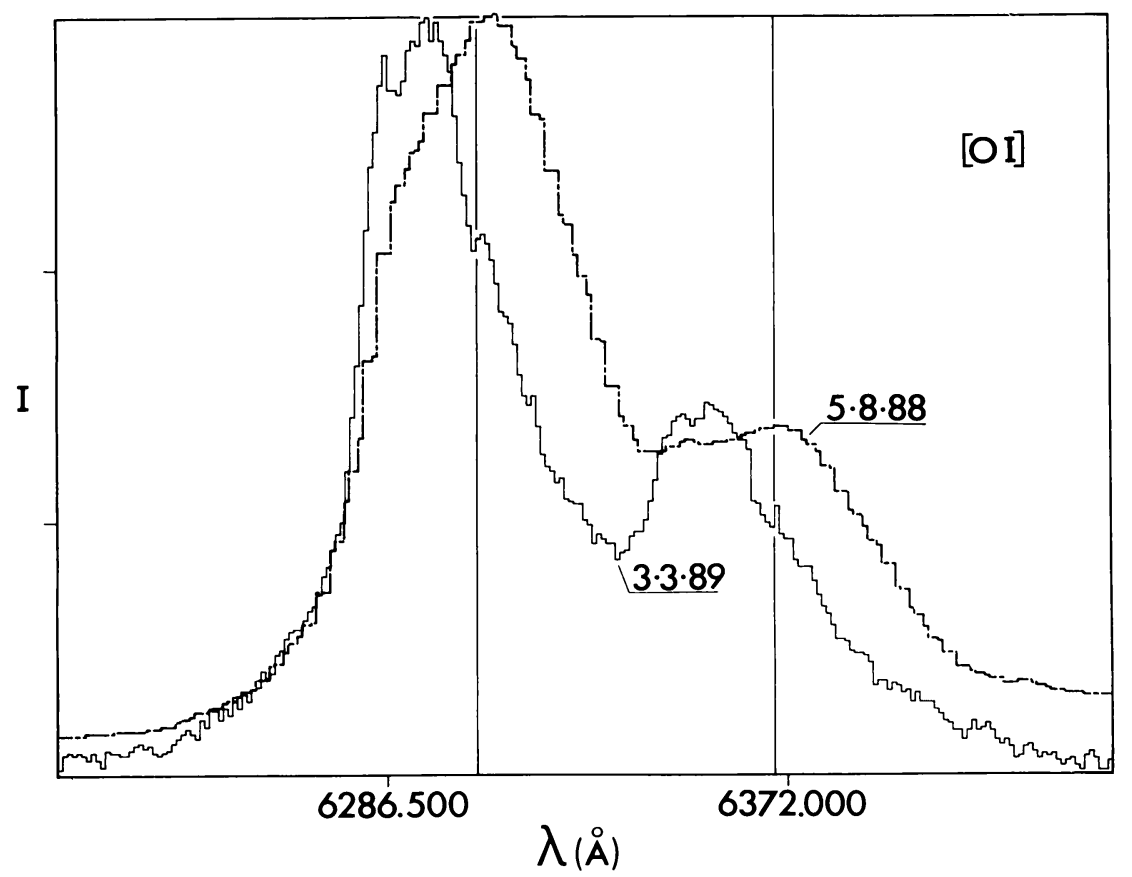

Fig.1. Profiles of [O I] 6300,63 lines before and after dust formation in the envelope of SN1987A.

determined from the spectroscopy when the dust began to form, when the rate of formation began to decrease, and when formation stopped. All of these events correspond to inflection points in the $\mathrm{V}$ light curve.

Thus, what has happened with dust in SN 1987A may be a common feature in supernovae. In fact, all previous cases of reported IR excesses may represent dust formation at different phases and therefore different temperatures in the envelopes. There is now an increasing number of instances of developing blue shifts in emission lines from supernovae. Turatto et al. (1989) have even conjectured on this possibility for the remnant of SN 1957d recently observed in M83.

How much dust has formed in SN 1987A? Unless and until the dust becomes optically thin and one can measure the characteristic resonance features for silicates near 10 and $20 \mu$, a direct determination of the mass is not possible. The fact that the Si I (+ [Fe II]) $1.644 \mu$ feature seemed to decrease in strength after day 530 faster than the extinction provided by either the amorphous or clumped dust suggests that this extra decrease could be caused by depletion of silicon onto grains. Lucy et al. (1990) suggest a possible formation efficiency $>50 \%$ which implies a mass of silicate grains in the vicinity of $0.1 \mathbf{M}_{\mathbf{*}}$. Whether this mass survives subsequent heating events in the remnant and finds its way into the interstellar medium remains to be seen. 


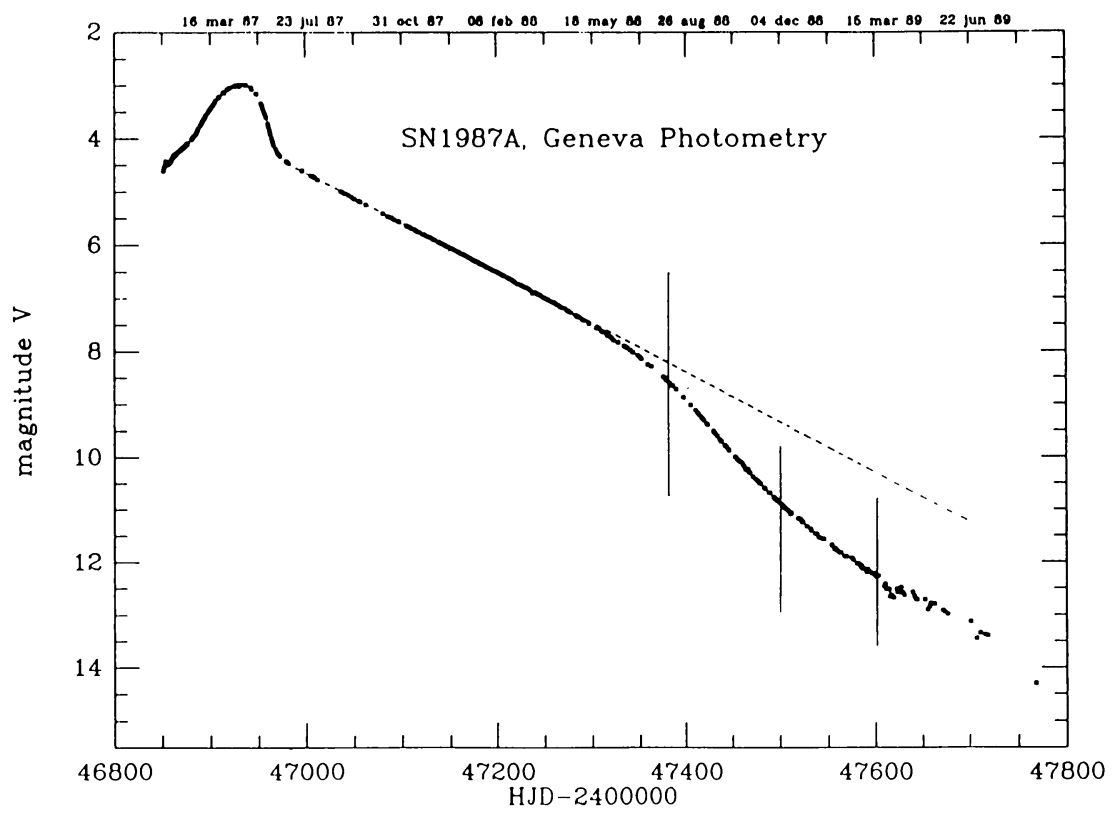

Fig.2. Geneva System V band photometry with vertical lines indicating dates marking various epochs in the dust formation process noted from the analysis of the line profiles.

\section{Line Luminosities and Profiles}

Apart from the systematic change of the profiles of emission lines signalling the formation of dust, the only other recorded changes in profiles of the broad lines have been those seen occurring irregularly and interpreted to be due to large scale inhomogeneities or clumping of material in the envelope. At the beginning of April 1990 (day 770) another significant change of profile occurred and persisted and is exemplified in Fig. 3 where profiles of the [OI] 6300.63 from different dates are overlain. It can be seen that a relative increase in flux on the blue side of the profile has occurred. It is not so obvious on the red side, but that observation may be hindered by the presence of dust obscuring more the far side of the envelope. This effect is also detectable in lines of $\mathrm{H} \alpha, \mathrm{Mg}$ I, $\mathrm{Na}$ ID.

At almost precisely the same epoch a lessening of the rate of decline of the luminosities of most emission lines was detected. The coincidence in time of the onset of these phenomena suggests a causal relationship. For simplicity of visualization, we propose the presence of two zones of material in the envelope, one of higher velocity and hotter, and one of lower velocity (probably on average, less extended) of lower temperature and undergoing the predicted IR catastrophe. It is the luminosity of this dominant cooler region which has been falling rapidly until, at day 770, the hotter higher velocity component becomes, by contrast, more obvious.

One further observation of interest is that the slope of the variation of the Si I $+[\mathrm{Fe} \mathrm{II]} 1.64 \mu$ feature does not flatten, suggesting, if our model is correct, that most of this radiation is coming from a cooler region. That the silicon and iron regions could be significantly cooler is qualitatively 


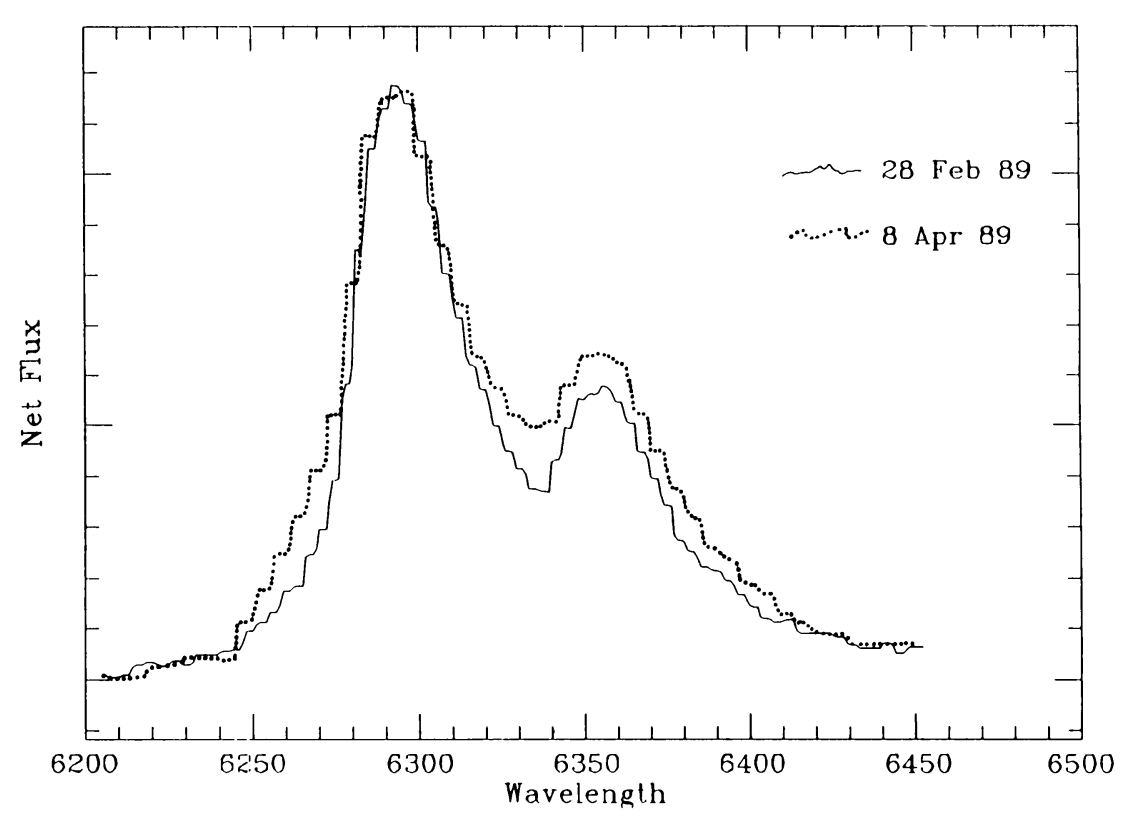

Fig. 3. Profiles of [O I] 6300,63 at the two epochs marked.

consistent with the fact that our preliminary calculations show that ions of $\mathrm{Si} \mathrm{I}, \mathrm{Fe} \mathrm{I}, \mathrm{Fe}$ II tend to have the highest ratios of radiative flux in emission lines per unit mass, and therefore in isolation can be cooling more rapidly. This is a simplification which needs elaboration in more quantitative detail.

\section{Cobalt Isotopes}

The radioactive energy source for powering the light curve is established theoretically and indirectly by observing the shape of the bolometric light curve after day 130 . It is known to be ${ }^{56}$ Co decaying from an initial mass of $\sim 0.075 \mathrm{M}_{\odot}$ with an e-folding time of 111.3 days. Its effect on the light curve might be expected to dominate to beyond 1200 days. Nevertheless if ${ }^{57} \mathrm{Co}$ is produced with an initial mass of $0.0017 \mathrm{M}_{\Theta}$, an amount suggested by the ratio of stable species $57 / 65$ measured in the solar system, the actual mass of ${ }^{57} \mathrm{Co}$ might exceed that of ${ }^{56} \mathrm{Co}$ after $\sim 500$ days. It does not dominate the light curve because the energy release from $\beta$-decay is less and the decay rate (e-folding time 390 days) slower.

By measuring emission lines of cobalt later than day 250 when the envelope has become optically thin, we can, in principle, determine the mass of cobalt present in all isotopic species. At ESO we have measured continuously the [Co II] $10.52 \mu$ line which arises from transitions within the fine structure levels of the ground state and whose strength therefore is insensitive to temperature. The results of these measurements have been compared with theoretical estimates based on assuming an initial production of $0.075 \mathrm{M}_{\odot}$ of ${ }^{56} \mathrm{Co}$ and also an initial $0.075 \mathrm{M}_{\bullet}$ of 
${ }^{56} \mathrm{Co}+0.0017 \mathrm{M}_{\Theta}$ of ${ }^{57} \mathrm{Co}$. There is some uncertainty due to the amount of unionized Co, but our assumed values come from estimates based on the measurements of emission lines from the first two ionization stages of $\mathrm{Ni}$ and $\mathrm{Fe}$ whose ionization potentials are similar to that of $\mathrm{Co}$.

Thus one deduces that a best fit to the data would, in the interval 400-520 days where discrimination can be made, suggest an initial value of $\sim 0.0028 \mathrm{M}_{\bullet}$ of ${ }^{57} \mathrm{Co}$, i.e. 1.5 times that suggested from the solar isotope ratios.

This value is consistent with a recent determination using the Co II $1.54 \mu$ by Varani et al. (1990) and the results of Oliva et al. (1989).

The value is consistent with the upper limit to the detection of the $147 \mathrm{keV} \gamma$-ray line from decay of ${ }^{57} \mathrm{Co}$ and with the limit on the X-ray flux resulting from the Comptonisation of this $\gamma$-ray line (Grebenev \& Sunyaev 1989) although uncertainties in modelling do now allow this to be a strong constraint. It is not inconsistent with the changes that have occurred in the bolometric light curve since day 900 . Note that the $10.52 \mu$ line suddenly dropped in strength after day 530 and this effect has been ascribed to the extinction by dust which began to form in copious quantities at that time (Danziger et al. 1989, Lucy et al. 1989). This also means that tracing the isotope ratios from that point on will really depend on very accurate measurement of the bolometric light curve.

\section{Chemical Abundances}

The early time modelling of the spectrum, particularly in the UV, by Lucy (1987) remains the most successful of these endeavours. Guided by abundance results obtained for the lighter elements in $H$ II regions Lucy used solar abundances $x 1 / 2.75$ for all elements to be representative of the chemical composition of the envelope. Details of the modelling may be obtained in that paper.

Recently Mazzali and Lucy (1990) have improved the treatment of excitation and ionization in these models. Prompted by the indications by Russell et al. (1988) and Russell and Bessell (1989) that the $\mathrm{Fe} / \mathrm{H}$ ratio may be half solar they have used this value for the iron group elements $(\mathrm{Mn}, \mathrm{Cr}$, $\mathrm{Fe}, \mathrm{Co}, \mathrm{Ni}$ ) and a value of one tenth solar for $\mathrm{C}, \mathrm{N}, \mathrm{O}, \mathrm{Mg}, \mathrm{Al}, \mathrm{Si}$ (from recent work of Lennon et al. 1990). The remaining elements continue to have a value of $1 / 2.75$ solar.

Two examples of models for days 1.60 and 1.85 illustrate a particular point. The newly adopted abundances for the iron group elements result in an improved fit to the absorption features ascribed to ions of those elements, and this sensitivity to varying abundance provides a reliable method for abundance determinations. The CNO elements play little rôle in contributing to absorption features in this part of the spectrum.

From the point of view of chemical evolution of the Large Magellanic Cloud these results are important. Equally important is an accurate determination of the chemical mix of elements produced by explosive nucleosynthesis and ejected into the interstellar medium. Preliminary results for stable isotopes have been presented by Danziger et al. (1990). These are at the moment necessarily relatively uncertain. In particular, the direct determination of the oxygen abundance suffers from large uncertainties.

Here we want to focus attention on the oxygen and iron abundances in galaxies such as the Magellanic Clouds.

Matteucci and Brocato (1990) and Matteucci (in preparation) have shown that because the Magellanic Clouds may have evolved more slowly than the solar neighbourhood and because iron is produced predominantly in Type I SN (with low mass progenitors), then the $\mathrm{O} / \mathrm{Fe}$ can be different from that in the solar neighbourhood at the present time (see Fig.4). This is a picture which is emerging with the observations of Russell et al. alluded to above. 


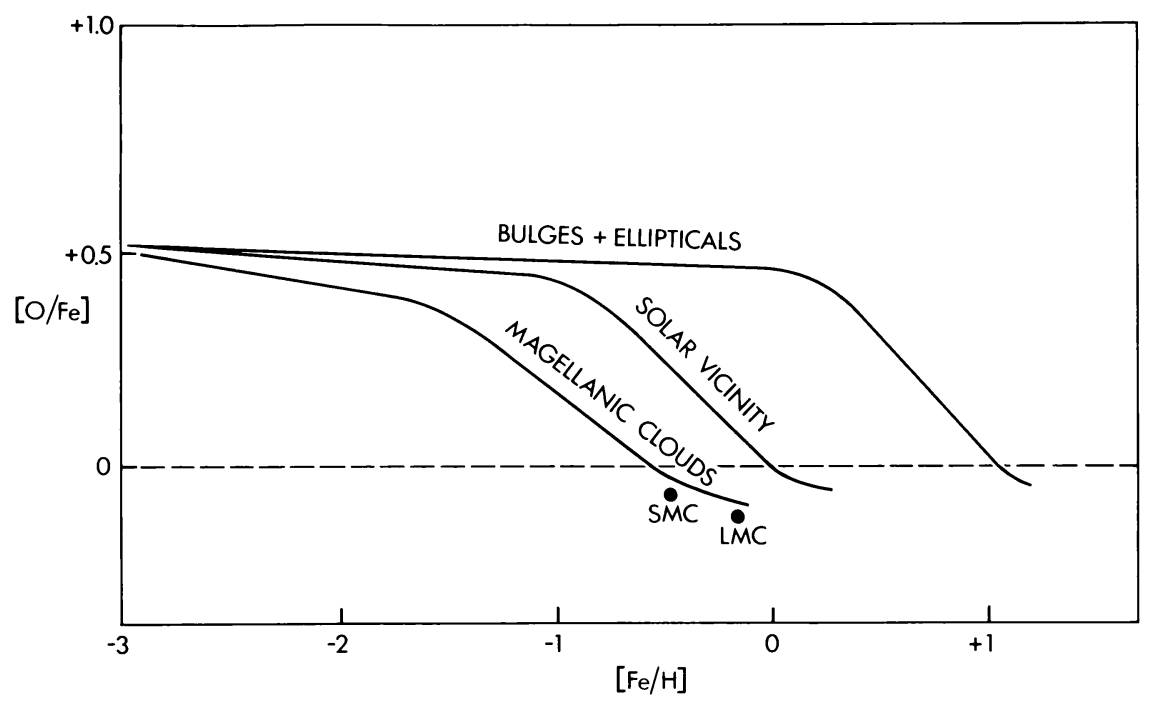

Fig. 4. Models of chemical evolution of galaxies from the work of Matteucci and Brocato (1990) and Matteucci (in preparation).

That there is an elegant explanation for these observations resulting from the time delay between Type II and Type I supernovae depends in the chemical evolution models upon theoretical yields from massive stars and oxygen in particular. For a star of $20 \mathrm{M}_{\Theta}$ (the progenitor of SN $1987 \mathrm{~A}$ ) the adopted yield is $1.5 \mathrm{M}_{\odot}$. Any significant deviation from that yield would directly affect the position of the lines in the figure representing the evolution of the Magellanic Clouds. That is one reason why it is important to narrow the range of uncertainty in the derived mass of oxygen in SN 1987A from the 0.2-3.9 $\mathrm{M}_{\boldsymbol{\theta}}$, where it sits at the moment.

\section{The recent Bolometric Light Curve}

There has been much interest in the bolometric light curve at late times ( $>900$ days) because of the possibility of new effective energy sources dominating the ${ }^{56} \mathrm{Co}$ input. Bouchet et al. (1989) announced that, starting at day 903 and extending to day 1030, a pronounced flattening had occurred. The degree of flattening did not seem as pronounced in the results of Suntzeff $e t$ al. (1990a,b) although some flattening was still implied. The reason for this difference is still not understood. The last two points in the ESO data suggest that the light curve is falling again more quickly although there remains an excess over what would be expected from radioactive decay of ${ }^{56} \mathrm{Co}$ alone. If one inspects the black-body fits to the IR excess where temperatures of $160^{\circ} \mathrm{K}$ are derived one sees that the bulk of the radiation now lies beyond the last ground-based measured point at $20 \mu$ (Q band). This must lead to uncertainties although experiments with forcing fits, still within the error bars of the observed points, show that the reported excess cannot be eliminated. Emission lines do not contribute an important component of the flux in the $\mathrm{Q}$ band at these late stages.

The hypothesis that a pulsar whose energy is thermalised by the dust seems now too simple, unless the dust in the past two months has become optically thin and the bulk of the radiation is 
escaping at higher energy. Measurement of the $\mathrm{N}_{1}, \mathrm{~N}_{2}, \mathrm{~N}_{3}$ bands around $10 \mu$ (designed for detecting the silicate emission feature from dust) have been too difficult because of the faintness and limited good weather conditions.

Matter falling onto an accretion disk surrounding a compact object at the centre has been an explanation proposed as a natural consequence of $10^{38} \mathrm{ergs} / \mathrm{sec}$ implied by the flattening (Woosley et al. 1989). That this accretion should stop just at the time at which we being to detect it seems an unlikely though not impossible coincidence. At this stage, with IR observations reaching the limit of current facilities, it is clearly advisable to search for other signs of energy input from harder radiation sources.

We are grateful to Francesca Matteucci for a discussion of her models of chemical evolution.

\section{References}

Bouchet, P., Danziger, I.J., Lucy, L.B. (1989) IAU Circular No. 4933.

Burki, G., Cromer, N., Nicolet, B. (1990) Astron. Astrophys. Suppl. in press

Danziger, I.J., Gouiffes, C., Bouchet, P., Lucy, L.B. (1989) IAU Circular. No. 4746

Danziger, I.J., Lucy, L.B., Bouchet, P., Gouiffes, C. (1990) Proceedings of Santa Cruz Workshop on Supernovae, Cambridge University Press, in press.

Dwek, E., et al. (1983) Astrophys. J. 274, 168

Graham, J.R., et al.(1983) Nature 304, 709

Grebenev, S.A., Sunyaev, R.A., (1989) Physics of Neutron Stars, eds D.A. Varshalovich, A.D.

Kaminker, G.G. Pavlov, D.G. Yakovlev, Nova Science Publishers, New York

Lennon, D.J. et al. (1990) Properties of Hot Luminous Stars, ed. C.D. Garmany, PASP, p.315

Lucy, L.B. (1987) Astron Astrophys. 182, L31.

Lucy, L.B., Danziger, I.J., Gouiffes, C., Bouchet, P. (1989) Proc. IAU Colloquium 120, Structure and Dynamics of ISM, eds. G. Tenorio-Tagle, M. Moles, J. Melnick. Lecture Notes in Physics, Springer-Verlag, p. 164.

Lucy, L.B., Danziger, I.J., Gouiffes, C., Bouchet, P. (1990) Proceedings of Santa Cruz Workshop on Supernovae, Cambridge University Press, in press

Matteucci, M. F., Brocato, E. (1990) Astrophys. J. in press

Mazzali, P., Lucy , L.B. (1990) IUE Conference, Toulouse, May, in press

Oliva, E., Moorwood, A., Danziger, I.J. (1989) IAU Symposium 132, ed. G. Cayrel de Strobel, M Spite (Kluwer, Dordrecht) p.601.

Russell, S.C., Bessell, M.S. (1989) Astrophys. J . Suppl. 70, 865

Suntzeff, N.B., Bouchet, P. (1990) Atron. J., 99, 650

Suntzeff, N., Phillips, M., Elias, J., Depoy, D., Walker, A. (1990a) IAU Circular. No. 4995

Suntzeff, N., Phillips, M., Elias, J., Depoy D., Walker, A. (1990b) IAU Circular. No. 4996

Turatto, M., Cappellaro, E., Danziger, I.J. (1989) The Messenger No. 56, 36.

Varani, G.-F., Meikle, W.P.S., Spyromilio, J., Allen, D.J. (1990) in press.

Woosley, S.E., Pinto, P.A., Hartmann, D. (1989) Astrophys.J. 346, 395 